\title{
Julius Hirschberg als Reisender durch Welt und Zeit
}

\section{Julius Hirschberg as a Traveller through Space and Time}

\begin{abstract}
„Auf unsrem von Waffen starrenden Planeten, dessen fernste Länder durch Dampfkraft und Electricität einander so nah gebracht sind während die Herzen der verschiedenen Völker leider noch nicht in gleicher Weise einander genähert werden konnten, fällt uns Männern der Wissenschaft die Aufgabe $z u$, nach dem Maß unsrer allerdings geringen Kräfte, für die friedliche Vereinigung der Völker Sorge zu tragen“. (Julius Hirschberg in der „3. Amerikafahrt“, 1905 [1])
\end{abstract}

Julius Hirschberg ( $\boldsymbol{O}$ Abb. 1) kann getrost zu den Titanen der Augenheilkunde gezählt werden. In intellektueller Hinsicht überragt er sogar seinen Lehrmeister $\mathrm{Al}$ brecht von Graefe (1828-1870). Kaum ein, ja höchstwahrscheinlich überhaupt kein anderer Ophthalmologe bisher hat ein so umfangreiches literarisches Werk - insgesamt mehr als 10000 Seiten als Alleinautor - hinterlassen wie Julius Hirschberg. Hirschberg wurde am 18. September 1843 in Potsdam geboren, wo er als einziger Sohn eines jüdischen Kaufmanns mit 4 Schwestern - 4 weitere Geschwister starben früh - aufwuchs [2-5]. In seinen späten Erinnerungen von 1923, deren 2. Teil leider nicht mehr erschien, beschrieb Hirschberg seinen Vater als "großen Geist und kleinen Kaufmann“ "2]. Ab 1853 besuchte er das königliche ViktoriaGymnasium in seiner Heimatstadt, das er 1862 als „Bester von allen“ verließ. Bereits in der Schule beschäftigte er sich intensiv mit Sprachen, Literatur und Geschichte $[2,3]$. Seine „ganze Wonne“ war die griechische Literatur. Vielleicht deshalb gestand er später, „dass ich als Tertianer die Mädchen gründlich verachtete, da sie kein Sterbens-Wort griechisch verstanden und auch sonst zu tieferer Unterhaltung mir wenigstens ziemlich unbrauchbar erschienen“ [2]. Als Sekundaner unternahm er, für damalige Verhältnisse sehr ungewöhnlich, ohne seine Eltern erste Reisen, die ihn in den Harz und nach Dresden, die sächsische Schweiz, Schlesien und Böhmen führten
[2]. Durch die Persönlichkeit des Hausarztes der Familie reifte in Hirschberg schon als Kind der Entschluss, Arzt zu werden [2]. Den Lebensunterhalt während des Medizinstudiums in Berlin musste er sich selbst als Hauslehrer und Stenograf der Berliner Medizinischen Gesellschaft verdienen $[2,4-6]$. Die Stenografie hatte er bereits als Jugendlicher erlernt. Sie sollte ihm später vor allem bei den Reiseberichten dienlich sein [2]. Besondere Hochachtung hatte Hirschberg während des Studiums vor dem Physiologen Emil du Bois-Reymond (1818-1896), dessen Sohn Claude später von ihm zum Augenarzt ausgebildet wurde. Augenheilkunde hörte Hirschberg bei dem seinerzeit schon als „veraltet“ geltenden Christian Jüngken (1793-1875) [2]. Hirschberg arbeitete während des Studiums ab 1864 als Famulus bei Rudolf Virchow (1821-1902), bei dem er viel mikroskopierte und 1866 mit einer Arbeit über die Placenta praevia promovierte $[2,6]$. In seiner Eigenschaft als Stadtverordneter sandte Virchow ihn 1866 an ein Cholera-Lazarett, das von Albrecht von Graefe geleitet wurde. So gelangte Hirschberg schließlich für 2 Jahre (1866-1868), noch vor dem Staatsexamen 1867, an die Graefe'sche Augenklinik und in die Ophthalmologie. 1868 ließ er sich als Augenarzt nieder. Trotzdem besuchte er an der Universität noch Vorlesungen über höhere Mathematik und, bei Hermann von Helmholtz (18211894), über Optik [2]. Nicht zuletzt aus diesem Grunde war er in der Lage, die Grundlagen der medizinischen Statistik zu formulieren [7-8] und sich mit der Dioptrik von Kugelflächen zu befassen [9], wie überhaupt aus vielen seiner Arbeiten (z. B. auch zur Strabologie) ein erstaunlich profundes mathematisches und physikalisches Wissen deutlich wird. Nachdem sich die finanzielle Situation gebessert hatte, unternahm er Studienreisen nach Wien, Prag, Paris und London [3], genau wie sein großes Vorbild Albrecht von Graefe. 1870 erfolgte die Habilitation, 1879 die außerordentliche Professur,

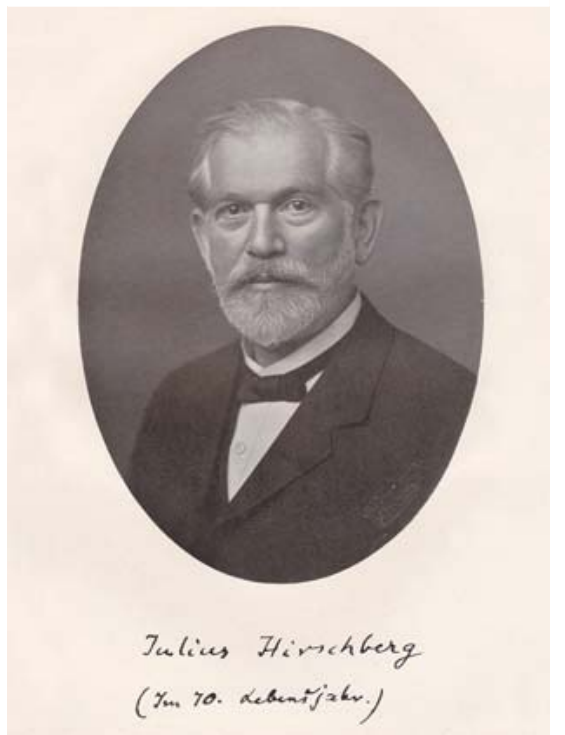

Abb. 1 Julius Hirschberg im 70. Lebensjahr, aus [11]

1895 die Ernennung zum „Geheimen Medizinalrat“. Im Auftrag des preußischen Kultusministeriums untersuchte Hirschberg 1896 und 1899 das Trachom in West- und Ostpreußen [10]. Im Jahre 1900 wurde er ordentlicher Honorarprofessor für Augenheilkunde an der Universität Berlin [10]. Das Berliner Ordinariat als Nachfolger von Carl Schweigger (1830-1905) im Jahre 1900 blieb ihm höchstwahrscheinlich aufgrund seiner jüdischen Herkunft, die bereits damals im Staatsdienst ein deutlicher „Wettbewerbsnachteil“" war, versagt, obwohl er sicher (mindestens) genauso qualifiziert war wie der dann berufene, gleichaltrige Julius von Michel (1843-1911) [5,6]. Hirschberg hat sich über den nicht an ihn ergangenen Ruf und über Antisemitismus niemals stärker beklagt. Eher beiläufig erwähnte er nur, dass „Antisemitismus damals (Anmerkung: in seiner Jugend) noch nicht erfunden war" und er aus dem „Akademischen Turnverein“ austrat, „als auch dieser später in das antisemitische Fahrwasser einlenkte“ [2]. Im Jahre 1893 gründete Hirschberg die Berliner augenärztliche Gesellschaft. Von 1897 bis 1903 war deren Präsident, danach deren Ehrenpräsident $[6,11] .1912$ erhielt er ein Ehrendoktorat der medizinischen Fakultät der Universität Athen. In internationalen augenärztlichen und wissenschaftlichen Gesellschaften aus Österreich, Frankreich, Belgien, Spanien, Italien, Ungarn, Russland, der Türkei, Brasilien, den USA und Ägypten war er korrespondierendes Mitglied [11]. Die Berliner 
Akademie der Wissenschaften verlieh ihm die Leibniz-Medaille in Silber [12]. Im Jahre 1869 hatte Hirschberg seine private Augenklinik mit Poliklinik - ganz nahe der Graefeschen - in der Berliner Karlstraße, heute Reinhardtstraße, mit 40 Betten eröffnet, über die er 1870 zum ersten Mal in der „Berliner klinischen Wochenschrift" unter Schilderung von Beobachtungen und Krankengeschichten berichtete [13]. In der Klinik etablierte er den weltweit wahrscheinlich ersten aseptischen Augenoperationsraum [12,14, 15]. Staatliche Hilfen nahm er selbst bei staatlichen Aufträgen, wie bei den Trachomuntersuchungen in West- und Ostpreußen, nicht in Anspruch [3]. Hirschberg schrieb dazu in seinen Lebenserinnerungen: „Unabhängigkeits-Sinn war das erste Zeichen von Charakter, das sich bei mir entwickelte. Auch in politischer Hinsicht habe ich freieren Anschauungen gehuldigt, von klein auf" [2]. Um bei dringenden Fällen schnell erreichbar zu sein, wohnte Hirschberg über 30 Jahre im Gebäude seiner Klinik [3,14]. Von 1869 bis 1907 behandelte er etwa 257000 Patienten und führte knapp 8000 größere Operationen selbst durch $[6,12]$. Sein jüdischer Schüler Wilhelm Mühsam (18741939) sagte posthum über Hirschberg als Arzt: „Bei der Vielseitigkeit seiner Interessen wusste er mit jedem seiner Patienten eine seelische Verbindung anzuknüpfen. Jeder hatte die Empfindung, dass er sich nicht nur für sein Leiden, sondern auch für seine Person wärmstens interessierte“ [3]. Der andere (jüdische) Schüler, Oskar Fehr (1871-1959), charakterisierte Hirschberg ähnlich: „Der Arztberuf war ihm eine heilige Mission, die Kranken ihm anvertrautes kostbares Gut, für die er jederzeit unermüdlich, selbstlos und opferfreudig seine ganze Kraft und sein großes Können eingesetzt hat. Seine Sorgfalt kannte keinen Unterschied zwischen Reich und Arm. Der Erfolg und der Dank der Kranken war ihm der schönste Lohn. Diese hohe Auffassung von unserem Beruf hat er seinen Assistenten eingeimpft fürs Leben. Die Freude an der Arbeit, das große Verantwortungsgefühl, der Respekt vor dem ihm anvertrauten Organ, die Gründlichkeit der Untersuchung, die Gewissenhaftigkeit bei der Indikationsstellung der Operation, die peinlichste Sorgfalt bei ihrer Vorbereitung und Ausführung, die Erwägung aller Möglichkeiten, die allein vor Überraschungen schützt, das sind Eigenschaften, die die Hirschbergschen Schüler täglich an ihrem Lehrer beobachten und bewun- dern konnten“ [4]. Auf der anderen Seite konnte Hirschberg, wie sein Schüler Harry Friedenwald (1864-1950) aus den USA berichtete, aber auch durchaus sehr harsch zu Patienten sein [12]. Hirschberg behandelte in seiner Klinik aber nicht nur Patienten, er übte auch eine umfangreiche Lehrtätigkeit aus. Wilhelm Mühsam sagte dazu: „Er, der selbst vier Erdteile bereist und, wie seine Reisebücher ,Um die Erde‘, ,Tunis' und 'Hellas-Fahrten' beweisen, mit Verständnis bereist hatte, sah in seiner Sprechstunde Bewohner aller Erdengegenden. Von allen Erdteilen kamen aber auch Fachgenossen, um ihn operieren zu sehen und seine Untersuchungsmethoden kennen zu lernen“ [3]. Unter den ausländischen Studenten stellten die US-Amerikaner offenbar eine sehr große Gruppe dar [1,12]. Hirschberg war polyglott, und seine Klinik war - wie die Graefe'sche zuvor - es bereits im 19. Jahrhundert ebenso.

Die augenärztliche Tätigkeit gab Hirschberg 1907 - nach seinen (wahrscheinlich inkorrekten) Angaben in den ,jungen Tagen“ 1909 - auf, um sich ganz der Fertigstellung der „Geschichte der Augenheilkunde“ widmen zu können $[2,4,10]$. Seine seit 1908 in einem Neubau an gleicher Stelle befindliche Klinik wurde von Wilhelm Mühsam weiter geführt, bis sie 1936 auf Druck der Nationalsozialisten geschlossen werden musste. Am Gebäude der ehemaligen Hirschberg'schen Klinik erinnert heute eine Gedenkplakette daran. Julius Hirschberg, der im Alter mal sagte „Ich habe keine großen Hoffnungen mehr, aber schöne Erinnerungen“, starb am 17. Februar 1925 in Berlin an einer Magenblutung. Er wurde auf dem jüdischen Friedhof zu Berlin-Weißensee beerdigt. Die Nachrufe seiner Schüler $[3,4$, 14] zeugen von tiefster Verehrung. Die 1986 gegründete deutschsprachige Vereinigung für die Geschichte der Augenheilkunde trägt den Namen „JuliusHirschberg-Gesellschaft“" (JHG).

Julius Hirschberg wird heute vor allem (und zu Recht) mit seiner monumentalen „Geschichte der Augenheilkunde“ verbunden, die er 1899 mit der „Augenheilkunde im alten Ägypten“ begann und 1918 mit dem 2. Teil der „Reform der Augenheilkunde“ abschloss. Die „Geschichte“ erschien in der 2. Auflage des „Handbuch der Augenheilkunde“ von Alfred Graefe (1830-1899) und Theodor Saemisch (1833-1909) und umfasst einschließlich Registerband etwa 4700 Seiten $[5,6,12,14]$. Vor allem wegen dieses Hauptwerks kann die Augenheilkunde von sich behaupten, von allen medizinischen Disziplinen am besten über die eigene Geschichte Bescheid zu wissen [5,6, 12]. Und doch gehen Hirschbergs literarische Leistungen, die er selbst [10], insbesondere aber seine Schüler zu seinem 70.Geburtstag zusammenstellten [11], sehr weit über die „Geschichte“ hinaus. Aufgrund der Tätigkeit bei Virchow und der „ophthalmopathologischen Neigungen“" Albrecht von Graefes [16] verwundert es nicht, dass seine erste Publikation überhaupt [17] und seine erste, den „Herren Professoren R. Virchow und A.v. Graefe“ gewidmete Monografie [18], beide über den „Markschwamm der Netzhaut“ (Retinoblastom), stark morphologisch geprägt waren. Auch später gab es immer wieder ophthalmopathologische Arbeiten. Hirschberg publizierte aber letztendlich zu fast allen Teilgebieten und sowohl zur konservativen als auch zur operativen Augenheilkunde. Dabei lag ihm, der er Berliner Krankenhäuser aufgesucht hatte um dort Patienten zu funduskopieren, vor allem die Thematik „Auge und Allgemeinerkrankungen“, wie z.B. Diabetes mellitus und Syphilis, am Herzen [3,6,11]. 1888 publizierte er als einer der Ersten, wenn nicht der Erste die tiefen Hornhautgefäße bei der syphilitischen Keratitis parenchymatosa [19]. Seit 1879 verwendete Hirschberg einen von ihm entworfenen, kleineren, daher handlichen Elektromagneten zur Entfernung intraokularer Fremdkörper [20-22]. 1885/ 86 beschrieb er den „Hirschberg-Test“ zur Schieldiagnostik $[23,24]$. Hirschberg gab lehrbuchartige Monografien [25], „klinische Beobachtungen“ [26], ein Wörterbuch der Augenheilkunde [27] und die Vorlesungen sowie mehrere Würdigungen seines heiß verehrten Lehrers Albrecht von Graefe, dem er auch den letzten Band seiner „Geschichte“ widmete, heraus [28-30]. Die „Vorlesungen Albrecht von Graefes 1859-1860“ von 1925 [31] waren Hirschbergs letzte Publikation. Graefes bis heute anhaltender Ruhm ist ganz maßgeblich seinem unermüdlichen Biografen und begabtesten Schüler Hirschberg zu verdanken [3]. Hirschberg beherrschte, wie es auch in der „Geschichte" und den Reiseberichten immer wieder anklingt, eine Vielzahl von Sprachen: Englisch, Französisch, Italienisch, Spanisch, Latein, (Alt-)Griechisch, Arabisch und wahrscheinlich auch etwas Hebräisch. So übersetzte er z.B. den „Aetius“ [32] aus dem Griechischen und den „Ibn Sina“ (Avicenna) [33] aus dem Arabischen. Er konnte sogar ägyptische Hie- 
roglyphen verstehen [12]. Im „deutschen Sprachverein“ hielt er 1892 einen Vortrag „Ueber die Sprache der Aerzte“, in dem er zahlreiche griechisch-lateinische Wortschöpfungen und eine auch dem Gebildeten unverständliche Sprache kritisierte und den Gebrauch der Muttersprache forderte [34].

Eine weitere „Großtat“ Hirschbergs war das „Centralblatt für praktische Augenheilkunde“, das er - von Graefe und Virchow nachahmend - 1877 begründete und bis 1919 - über mehr als 40 Jahre - als Schriftleiter betreute. Das bei Veit \& Comp. (Compagnie) erschienene „Centralblatt“ brachte neben Originalarbeiten vor allem auch Rezensionen zu Publikationen ophthalmologischen Inhalts. Es bot Hirschberg eine Plattform für seine eigenen, unzähligen Veröffentlichungen. So fanden z.B. mehr als 75 „Nekrologe“ (Nachrufe) und ungewöhnlichere Abhandlungen wie etwa zur vergleichenden Ophthalmoskopie Hirschberg spiegelte den Augenhintergrund u. a. von Frosch, Salamander, Schildkröte, Alligator und Python [35] - oder zur Dioptrik des Fisch- und Froschauges [36] in Kurzform ihren Platz im „Centralblatt“. Das Herausgebergremium war zu Hirschbergs Zeiten vermutlich nicht zuletzt aufgrund seiner Reisetätigkeit international besetzt. Es umfasste aber vor allem einen hohen Anteil jüdischer Fachkollegen. So lag deren Anteil beispielsweise 1913 bei 30\% (Anteil der Juden an der Gesamtbevölkerung seinerzeit ca. $1 \%$, [Augen-]Ärzteschaft ca. 10 $15 \%)$, sodass von einer gewissen ,jüdischen Verbundenheit" in der Ophthalmologie des Deutschen Kaiserreichs ausgegangen werden kann. Im „Editorial Board“ 1913 befanden sich u. a. Oskar Fehr, der 1939 nach Großbritannien emigrierte, und Wilhelm Mühsam - beide gaben die Festschrift zum 70. Geburtstag Hirschbergs heraus [11] - aber z.B. auch Arthur Czellitzer (geb. 1871) aus Berlin, der 1943 im KZ Sobibor ums Leben kam, oder Sigmund Neuburger (geb. 1867) aus Nürnberg, der 1936 Selbstmord beging [37,38]. Das „Centralblatt“ wurde später, nachdem Hirschberg selbst keinen Nachfolger finden konnte, im Berliner Springer-Verlag als „Zentralblatt für die gesamte Ophthalmologie und ihre Grenzgebiete" bis 1996 fortgeführt. Über das „Centralblatt“ hinaus war Hirschberg neben Hermann Knapp (1832-1911) eine Zeit lang als Herausgeber des „Archiv für Augenheilkunde“ tätig ( Abb.2). Ganz egal wo und was er publizierte, galt für ihn immer der Grundsatz „Augenheilkunde ist weder eine Gewebelehre des Auges noch eine mathematische Formelsammlung, noch eine Pilzkunde; sondern die wissenschaftliche Darstellung der Kunst, kranke Augen zu heilen und gesunde so zu erhalten. Drei Dinge muss derjenige kennen lernen, welcher Augenkrankheiten zu heilen unternimmt: die Augenheilmittel, das Auge, die Augenkrankheiten“ [25].

Verständlicherweise blieb Julius Hirschberg in seinen wissenschaftlichen Publikationen nüchtern-sachlich. In der „Geschichte“ ist er als Autor wahrzunehmen, der um Objektivität bemüht ist, immer wieder großes Lob ausspricht, aber oft auch mit persönlicher Kritik, ja abfälligen Bemerkungen insbesondere gegenüber anderen Medizinhistorikern nicht spart. So kommt selbst der jüdische Glaubensgenosse und Graefe-Mitschüler Hermann Cohn (1838-1906) trotz der Anerkennung seiner Leistungen bei Hirschberg nicht ausnahmslos gut weg [39]. Der „Mensch Hirschberg“ erschließt sich im „Centralblatt“, besser noch in den Nachrufen seiner Schüler [3,4,14], am besten aber in den zwischen 1885 und 1910 entstandenen Reiseberichten.

Das 19. Jahrhundert brachte eine erhebliche Verbesserung der Verkehrsmittel und damit eine Erhöhung der Mobilität. Es war dabei nicht unüblich, darüber zu berichten. So schilderte Albrecht von Graefe seine Reisen in den Briefen an Frans Cornelis Donders (1818-1889) [40]. Julius Jacobson (1828-1889) aus Königsberg, Lieblingsschüler und, nach dessen Aussage, Gewissen Albrecht von Graefes, schrieb Reisebriefe aus der Schweiz und aus Italien, die posthum veröffentlicht wurden [41]. Hirschberg konnte und wollte sehr viel reisen, denn für ihn war „die einzige wirkliche Quelle für die Beschreibung die eigene Anschauung“ [42]. 1923 konnte er auf 73 Reisen zurückblicken und dazu feststellen, „dass nächst der Schule und der Universität sowie dem eignen Studium nichts anderes soviel zu meiner Ausbildung beigetragen hat als gerade das Reisen“ [2]. Neben vielen anderen, zum Teil bereits genannten Destinationen besuchte er Paris, Rom, Sizilien, Utrecht, London, Glasgow, Edinburgh, Cork/Irland, Bulgarien, die Schweiz und Kleinasien, ja sogar, 1895, Spitzbergen [1,11,43,44]. 1905 berichtete er in 4 Teilen über „Meine dritte Amerika-Fahrt“ in der „Medizinischen Klinik“ und darin über die seinerzeitigen Verhältnisse in der US-amerikanischen Ophthalmologie. Die Reise führte ihn von New York über Chicago nach Portland/ Oregon, wo er bei der „Sektion Ophthal- mologie“ der „American Medical Association“ einen eingeladenen Vortrag über „Die Geschichte der Augenheilkunde bei den Arabern" hielt, und zwar bewusst „ohne die geringste Beziehung zur Praxis, zur Krankenheilung, zum Gelderwerb, sondern nur zur Befriedigung des wissenschaftlichen Sinns der Gebildeten“. Die Resonanz auf den Vortrag war sehr positiv, und Hirschberg wurde zum Ehrenmitglied der Gesellschaft ernannt. Die Rückreise führte ihn über San Francisco, Los Angeles, Denver, Chicago und Boston zurück nach New York [1].

Über mehrere kurze Berichte und die ausführlichere „3. Amerikafahrt“ hinaus verfasste Hirschberg 5 große Reisemonografien, nämlich:

1. „Eine Woche in Tunis“ von 1885, 113 Seiten [45]. Die Reise wurde 1884 mit der Ehefrau unternommen, der er auch die Monografie widmete. Ziele waren vor allem Tunis und Karthago.

2. „Von New York nach San Francisco“ von 1888, 276 Seiten [42]. Die Widmung lautete „Seinem Freunde H. Knapp in New York zur freundlichen Erinnerung“. Passend zum Reiseziel verwendete Hirschberg im Titel „M.D.“ statt „Dr.“ ( Abb.3). Die Reise führte von New York über Chicago, St. Paul, den Yellowstone-Park und Portland nach San Francisco und von dort, nach Abstecher zum Yosemite-Tal, über Salt Lake City, Denver, Chicago und Niagara wieder zurück nach New York.

3. „Aegypten - Geschichtliche Studien eines Augenarztes“ von 1889, 116 Seiten [46]. Die Abhandlung ohne Widmung besteht aus 3 Teilen. Teil I („Aegypten als klimatischer Curort“) kann als „Reiseführer mit eigenen Erfahrungen“ bezeichnet werden. Hirschberg bereiste - wahrscheinlich ohne seine Frau, jedenfalls wird diese nicht erwähnt - vor allem Kairo, Alexandria und Luxor. Teil II „Ueber die Augenheilkunde der alten Aegypter“ befasste sich zum großen Teil mit dem „Papyrus Ebers“, auf dem die alte ägyptische Heilkunde, allerdings nur als Krankheits- und Rezeptsammlung, überliefert ist. Mit dem Entdecker des Papyrus, Georg Ebers (1837-1898), seit 1870 außerordentlicher, ab 1875 ordentlicher Professor für Ägyptologie in Leipzig, stand Hirschberg, wie er selbst schreibt, in enger Korrespondenz zwecks Interpretation der alten (ophthalmologischen) Heilmethoden. Teil III („Ueber die ägyptische Augenent- 


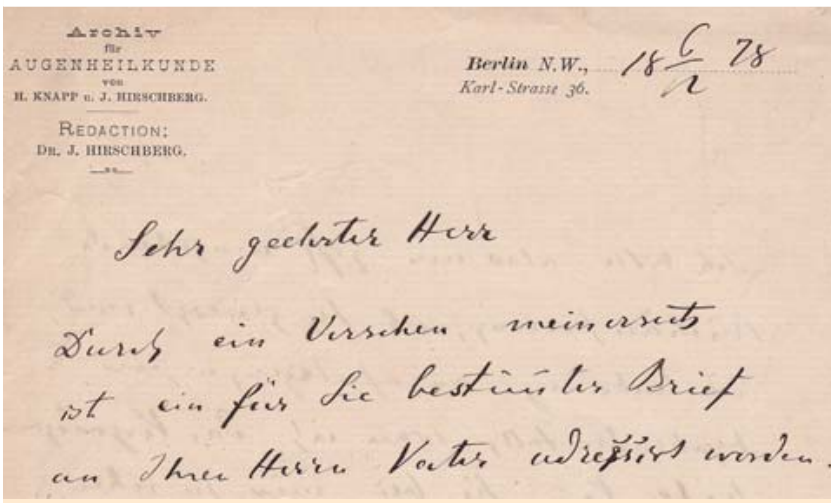

Abb. 2 Originaler Brief Hirschbergs vom 6. Dezember 1878. Im Briefkopf firmiert er unter der Adresse seiner Klinik (Karl-Strasse) als Herausgeber des „Archiv für Augenheilkunde“ gemeinsam mit Hermann Knapp, der ebenfalls - früher als Hirschberg - bei von Graefe gearbeitet hatte. Hirschberg hatte offenbar die alleinige Redaktion der Zeitschrift inne, nachdem Knapp bereits 10 Jahre zuvor, 1868, in die USA ausgewandert war (Archiv des Autors).

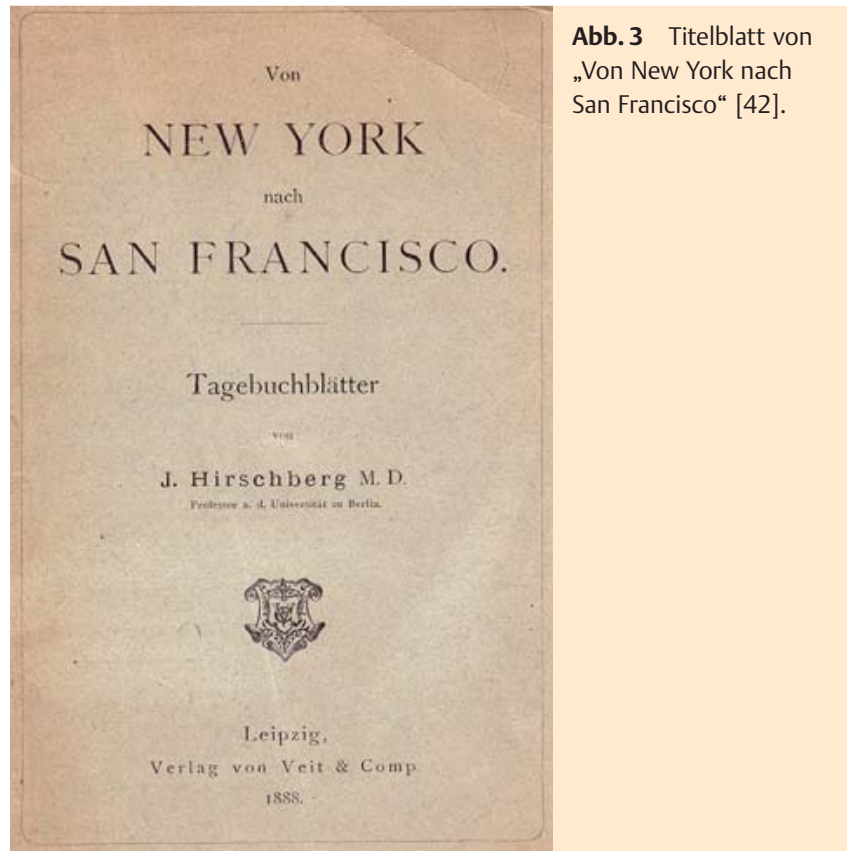

zündung“) schließlich beschäftigte sich mit der Geschichte des Trachoms.

4. „Um die Erde“ von 1894, 531 Seiten [47]. Auch dieses Buch ist der Ehefrau gewidmet, welche die Reise aber nicht mitmachte. Es ist von allen das wohl spannendste, auf jeden Fall das mit Abstand umfangreichste. Als einziges enthält es wenige Abbildungen ( $\bullet$ Abb.4). Eine Weltkarte zeigt den Reiseverlauf ( Abb. 5). Über die (augen-) ärztlichen Aspekte der Reise, insbesondere die Besuche von Fachkollegen und Krankenhäusern, berichtete Hirschberg separat in mehreren Beiträgen für die „Deutsche Medicinische Wochenschrift" [48].

5. „Hellas-Fahrten“ von 1910, 264 Seiten [49]. Das Buch, das der 1908 verstorbenen Ehefrau gewidmet ist, geht auf 3 Griechenlandreisen in den Jahren 1886, 1890 und 1909 zurück. Bei den ersten beiden Reisen war die Ehefrau dabei. Die letzte war die ausgedehnteste, die Hirschberg zu fast allen historisch bedeutsamen Stätten führte.

Hirschberg machte sich auf den Reisen stenografische Notizen, die Publikation der Monografien erfolgte ausnahmslos im Jahr nach der Reise, also zeitnah. Bei den großen Reisen führte er die Entfernungen der Teilstrecken dezidiert auf ( Abb. 6). Drei der 5 Reiseberichte erschienen (wie die Mehrzahl seiner Monografien) bei Veit \& Comp. in Leipzig, wofür das dort herausgegebene „Centralblatt“ ausschlaggebend gewesen sein dürfte. Alle Reisen wurden unter Hinzuziehung der damals einschlägigen deutschen und englischsprachigen Literatur sehr gründlich geplant und vorbereitet [6]. $\mathrm{Zu}$ wesentlichen organisatorischen Pannen oder Verzögerungen während der Reisen kam es daher, etwas überraschend, offenbar nicht.

Alle Berichte spiegeln Hirschbergs außergewöhnliche Kenntnisse bezüglich Geschichte, Geografie, Geologie, Wirtschaft, Politik, Kunst, Kultur und Religionen(en) der bereisten Länder wider, wobei Japan, Indien und Ceylon (heute Sri Lanka), der „Traum seiner Jugend“ [47], es ihm augenscheinlich besonders angetan hatten. Sehr ausführlich beschrieb Hirschberg die Verkehrsmittel, die er benutzte. Von den Dampfschiffen kannte er Länge, Wasserverdrängung und PS der Dampfmaschine, von den Eisenbahnen die Spurweite. Stets gab er akribisch die Fahrpreise, die Reisedauer und die Geschwindigkeit an. Hirschberg reiste in der Bahn „1. Klasse" und auf dem Schiff "Kajüte“ (Oberdeck). Sollte er die Kajüte teilen, konnte er durchaus energisch werden: „Der Versuch, mir sofort einen Cajütgenossen zu geben, schlug ich siegreich zurück, erst mit Güte, und dann, als dieses nicht half, mit Grobheit. Als ältester Reisender des Dampfers, der noch dazu die ganze Fahrt (Anmerkung: von Hongkong nach Colombo) mitmachte, glaubte ich dieses Vorrecht zu verdienen“ [47]. Wenngleich er auch mancherorts sehr einfache Herbergen aufsuchen musste, so versuchte er doch, in den besten Hotels am Ort unterzukommen. Trinkgelder verteilte er großzügig. Die Währungs- beschaffung im Ausland bereitete ihm keine Probleme. Ganz offensichtlich war Hirschberg dank seiner Augenklinik vermögend. Er führte ein „Aneroid-Barometer" mit sich, sodass er Temperatur und Höhe über dem Meeresspiegel protokollieren konnte.

In den Reiseberichten finden Angehörige, wie z.B. die Geschwister, keinerlei Erwähnung. Kinder blieben Hirschberg und seiner Gattin zu seinem großen Kummer versagt $[4,6]$. Nur zu seiner von ihm innig geliebten Frau, die ihn auf einigen Reisen begleitete und das „größte Glück seines Lebens“ war [3], bemerkte er beiläufig, dass sie eine etwas schwächliche Konstitution hatte und zur Seekrankheit neigte [45]. Demgegenüber war Hirschberg robust und bis ins hohe Alter gesund $[3,4$, $6,12,14]$. Tagelange Wanderungen durch unwegsame Landschaften und auf höhere Berge - man würde das heute als „Trekking-Touren“ bezeichnen - machten ihm nichts aus, selbst unter den tropischen Bedingungen Ceylons oder, in Griechenland, bereits in höherem Alter (von 66 Jahren) $[47,49]$. Und so ist er dann auf den ganzen Reisen offenbar niemals ernstlich krank geworden. Hirschberg schrieb dazu: „Jedenfalls bin ich gesund geblieben, hier (Anmerkung: in Colombo) und auf der ganzen Reise. Weitere Arzneien habe ich nicht gebraucht, nur die in meiner Reiseapotheke mitgenommenen Carbolsäurepastillen, um in Colombo die weiße Schimmelkrankheit meines Fracks zu beseitigen“ [47]. Mag hieraus ein gewisser Witz sprechen, so 


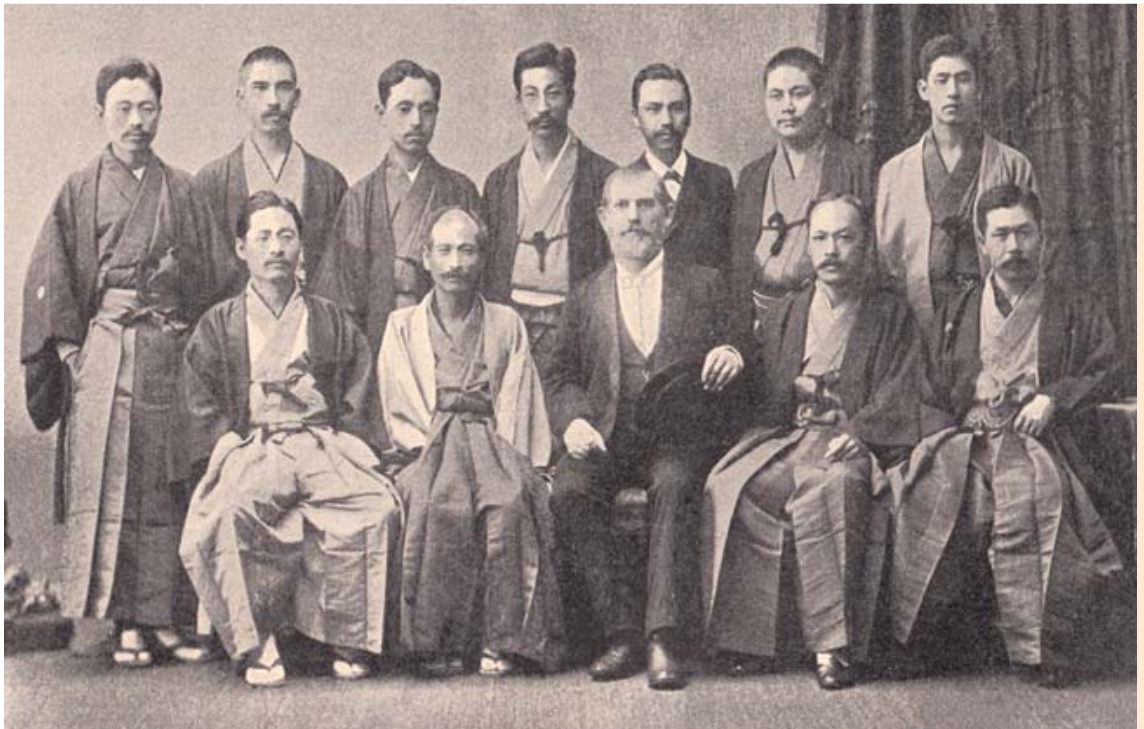

Abb. 4 Julius Hirschberg mit „Begrüßungskomitee“ von Tokyoter Augenärzten, September 1892. Höchstwahrscheinlich war einer der japanischen Kollegen Jujiro Komoto, aus [47]

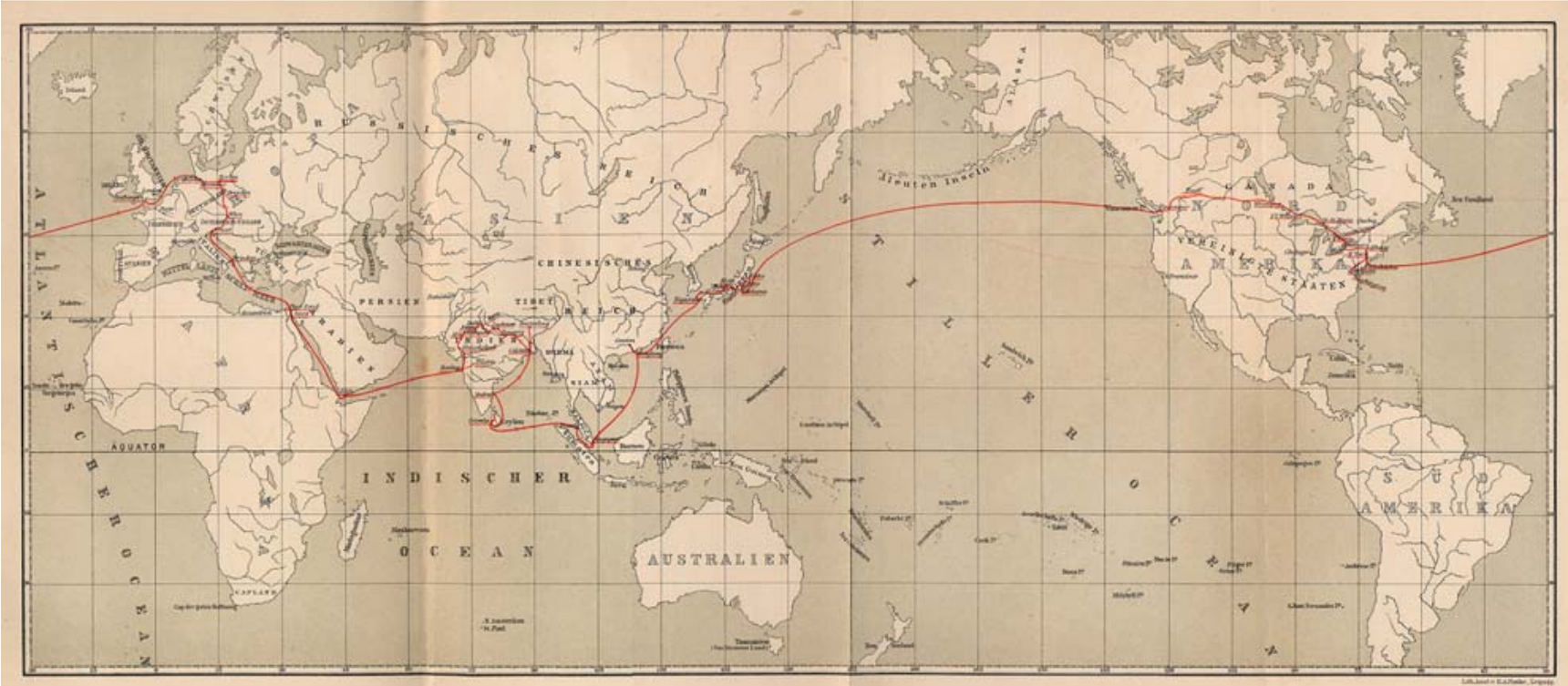

Abb. 5 Weltkarte mit rot eingezeichneter Reiseroute. Hirschberg startete in Berlin nach Westen und bestieg das erste Schiff in Bremerhaven, aus [47].

galt Hirschberg bei seinen Schülern doch als humorlos: „Der Meister der Rede war auch ein unvergleichlicher Plauderer. [...] Für Humor hatte er wenig Verständnis, und ein Scherz musste schon von Aristophanes oder von Shakespeare sein, um Gnade vor seinem Ohr zu finden“ [3] und „He hardly ever laughed, except when he made a sarcastic remark about one of his colleagues" [6]. Dementsprechend sind auch die Reiseberichte nicht eben gerade mit Humor gespickt, und doch bringen einige Sätze den heutigen Leser zum Schmunzeln wie „Den Europäer fesseln Beweisstücke der erstaunlichen Fresswerk-Leistungen einheimischer Ameisen (Anmerkung: auf Ceylon), wie mannsdicke Balken, die in eine Art Flechtwerk umgewandelt sind [...]; man würde sich kaum noch über durchgefressene Eisenbahnschienen verwundern“, „Die Gesänge mehrerer Matrosen mussten sogar als Geheul bezeichnet werden“ oder „Der Japaner ist im Räuschchen nicht unliebenswürdig“" [47].

Hirschberg war durchaus Genießer und alles andere als abstinent. Gutes, regionales Essen gehörte zu den Reisen genauso dazu wie der Genuss von Zigarren. So schrieb er etwa „Bis Yokohama hatten meine Havannas gereicht“, „Die durch den Regen erfrischten Blüthenbäume auf der Strasse mischen ihren Duft mit dem meiner Morgen-Cigarre“ oder „Da ich bei
Tage nicht gern schlafe, hilft mir eine indische Cigarre und ortsangemessener Lesestoff, die Zeit zu vertreiben“ [47]. Hirschberg trank, vor allem in Griechenland, aber sogar auch in Japan gern Wein, auf Ceylon bereitete er sich einen heißen Grog, und gegen Seekrankheit empfahl er einen Cognac. Immer wieder musste es aber, egal an welchem Teil der Erde er sich befand, ein „gutes Bier“ sein. „Das Frühstück schmeckte vortrefflich, ebenso ein Fläschchen Pilsner Bier und ein Mittagsschläfchen, das ich ausnahmsweise, nach dem Spaziergang in den Tropen (Anmerkung: auf Ceylon), mir gönnte“ [47]. Aus Südkalifornien berichtete Hirschberg: „Doch gelang es mir eine Flasche 


\section{Entfernungen :}

1. Berlin-Bremerhafen.

2. Bremerhafen-New-York

3. New-York, Washington, Baltimore, Philadelphia, New-York .

4. New-York-Albany

5. Albany-Niagara-Owen Sound . . .

6. Owen Sound-Fort William

7. Fort William-Vancouver

8. Vancouver-Yokohama

9. In Japan bis Kobe

10. Kobe-Hongkong .

11. Hongkong-Colombo

12. In Ceylon

13. Colombo-Calcutta

14. In Indien

Calcutta-Darjeeling und zurūck 758 E. M.

Calcutta-Benares . . . . 476 ,.

Benares-Lucknow . . . 200 ,

Lucknow-Cawnpur. . . 35 ”

Cawnpur-Agra . . . . 240 ,

Agra-Delhi . . . . . 243

Delhi-Bombay . . . . . 890

Bombay-Ellora und zurück 468

Bombay-Triest .

3310 E. M. 4367 See-M.

5330.

8082.

1390.

48092.

Abb. 6 Zusammenfassung der Weltreise mit Kilometerangaben, aus [47].

456 Engl.M. 753 .

143 E. M. 229.

486 E. M. 782.

555 See-M. $\quad 893$.

1900 E. M. 3058.

4283 See-II. 7744.

614 E. M. 987.

1367 See-M. 2637.

3096 See-M. 5875.

444 E. M. 714.

1380 See-II. 2550.

Triest-Wien-Berlin . ge', sagte er, ,hier hast du sechs Dreier. Die bekommst Du, wenn die Sache dir zusagt, für jeden Tag, monatlich vorausbezahlt. Du gehst hinüber in das anständige Bierhaus, wo nur unsre guten Bürger verkehren. Spricht einer von den Bekannten mit dir, so antwortest du, wie es sich gehört; wo nicht, so schweigst du. Punkt neun Uhr bist du wieder zu Hause und übersetzest mir aus dem Griechischen, zuerst den Homer, bis 10 Uhr، “ [2].

Zu seinem Vaterland Deutschland hatte Julius Hirschberg bis zuletzt ein sehr inniges Verhältnis, obwohl der Staat ihn bei der Besetzung des Berliner Lehrstuhls 1900 diskriminierte. Seine Adresse zum Ausbruch des Ersten Weltkriegs ( $\triangle$ Abb. 7) [50] lässt ihn als „Nationalisten“ erschei- nen, doch war er zuvorderst ein Sohn seiner Zeit; nicht mehr, und nicht weniger. „Eine warme Vaterlandsliebe beseelte ihn. Politisch hielt er sich zurück, sein Deutschtum aber hat er hochgehalten, wo immer er weilte“, befand Oskar Fehr im Nachruf [4]. Und Wilhelm Mühsam ergänzte: „Um so tiefer empfand er es, dass der Krieg die völkerverbindende Brücke der Kultur zerstörte, noch tiefer aber, dass sein Ausgang den Zusammenbruch des von ihm heiß geliebten Vaterlandes bedeutete" [3].

Hirschbergs Patriotismus wird auch dadurch deutlich, dass er in der Absicht, dem Staat Finanzmittel zu gewähren, einen sehr großen Teil seines Vermögens während des Ersten Weltkriegs in Kriegsanleihen anlegte und schließlich verlor, was ihn zwang, seine überaus umfangreiche, ca. 3200 Bücher, 12500 Sonderdrucke sowie historische Dokumente umfassende Privatbibliothek mit auf Deutsch geschriebenem Brief vom 2. Dezember 1920 Jujiro Komoto (1859-1938), dem ersten Professor für Augenheilkunde an der Universität Tokyo ab 1890, für 40000 Yen in 4 Raten à 10000 Yen anzubieten $[6,12,51]$. Komoto, der in den 80er-Jahren des 19. Jahrhunderts für einige Zeit Schüler Hirschbergs in Berlin gewesen war, und den Hirschberg 1892 auf seiner Weltreise besucht hatte, ging auf das Angebot ein [51]. Wegen des Todes Hirschbergs wurde die letzte Rate nicht mehr bezahlt. Die Bibliothek wurde als „Komoto-Bibliothek (früher Hirschberg-Bibliothek)“ Teil der Zentralbibliothek der Universität Tokyo und 1938 rekatalogisiert $[12,51]$. In Berlin war sie „nach Meldung den Fachgenossen zugänglich“ und hätte eigentlich, so Hirschberg im Vorwort zum „Katalog“ 1901, „dereinst durch Schenkung in den Besitz der Berliner med. Gesellschaft übergehen“ sollen [52].

\section{Fürs Vaterland.}

Seitdem das Juli-Heft unsres Centralblattes nach dem In- und Ausland versandt worden, ist der furchtbare Krieg ausgebrochen, den unsre Feinde ruchlos über uns gebracht.

Bei unsren siegreichen Heeren wirken als Stabsärzte viele von unsren Mitarbeitern, so Dr. F. Mender, Dr. W. MÜHSAM, Dr. SpIRo, alle aus Berlin.

Wir Alten, denen die Jahre den Dienst an der Front leider nicht mehr gestatten, versuchen daheim unsre Pflicht zu tun, in erster Linie für die verwundeten Krieger, für die bedrängten Bürger; sodann aber auch für die Wissenschaft, auf deren Altar das ewige Feuer zu unterhalten gleichfalls eine Pflicht gegen unser geliebtes Vaterland darstellt.

Berlin, am 28. August.

xxxviII
Julius Hirschberg.

15
Abb. 7 Adresse Julius Hirschbergs zum Ausbruch des Ersten Weltkriegs im August 1914, aus [50]. 
Wahrscheinlich fand Hirschberg angesichts der beginnenden Geldentwertung im Deutschen Reich keinen inländischen Abnehmer für seine Bücher. (Deren Rückführung nach Deutschland wäre wünschenswert. Deutsch war seinerzeit noch Wissenschaftssprache und auch in Japan geläufig, sodass die Bibliothek in Tokyo nutzbar war. Heute dürfte das nur noch höchst bedingt der Fall sein.) Der Verlust der Bibliothek muss Hirschberg zutiefst getroffen haben. Er hatte aber, was den Ersten Weltkrieg angeht, bereits 30 Jahre vorher, 1885, eine Ahnung gehabt, als er feststellte, dass „die Entfesselung der nationalen Leidenschaften vielleicht das Schlimmste ist, was Epimetheus-Napoleon aus seiner Pandorabüchse uns hinterlassen hat" [45]. Galt den Indern, Chinesen und, ganz vorn, den Japanern, die er als „fröhlicher, vielleicht glücklicher, als wir" charakterisierte [47], seine Sympathie, so war sein Verhältnis vor allem zu den Engländern, auf die er bei seinen Reisen naturgemäß immer wieder traf Indien und Ceylon waren seinerzeit britisches Protektorat - ambivalent. Wenngleich er einige Leistungen der Engländer wie die Organisation der Verwaltung in Indien oder die wirtschaftliche Entwicklung Ceylons durchaus anerkannte, konnte er sich mit den kolonialen Bestrebungen Britanniens nicht anfreunden. Man hat sich dabei in Erinnerung zu rufen, dass zwischen England und dem Deutschen Reich nach der Reichsgründung 1871 zunehmend Rivalität aufkam. Ausführungen wie „Das Mittagessen im Oriental-Hotel (Anmerkung: in Colombo) trägt die ganze Wichtigthuerei und geheuchelte Vornehmheit zur Schau, die jeder kennt, der im Alexandra-Hotel zu Oban in Schottland oder in Shepheard's Hotel zu Cairo in Ägypten unter überwiegend englischer Gesellschaft zu speisen das Vergnügen gehabt“, „Da war (Anmerkung: in Colombo) ein englischer Beamter, der bei Tisch die abfälligsten Aeusserungen über mein Vaterland sich erlaubte und nachdrücklich zum Schweigen gebracht werden musste. Selbst einer meiner alten Reisegefährten von der Empress (Anmerkungen: Name eines Schiffs; Hirschberg traf während seiner Weltreise immer wieder auf Reisende, die er von früheren Schiffspassagen her kannte), ein Gymnasialprofessor, trieb es ebenso; wurde aber weit angenehmer, als ich mir nichts gefallen liess, sondern sofort zum Angriff auf englische Zustände vorging“ und schließlich „,Geben Sie doch ElsassLothringen an die Franzosen', sagte am ersten Abend in Bombay mein zufälliger Tischnachbar, ein Brite, nachdem er meine Herkunft erkundigt. ,Geben Sie', erwiderte ich, ,Gibraltar, Malta, Cypern, Aegypten an ihre Eigenthümer, und keinen Rath an diejenigen, die ihn nicht wollen“ “ [47] lassen darauf schließen, dass bei Hirschberg keine besondere Anglophilie bestand. Dennoch pflegte er freundschaftlichen Kontakt auch zu britischen Kollegen, und 1904 war er Ehrengast der britischen Ärztegesellschaft in Oxford [1]. Über die USA schrieb Hirschberg: „Der Eindruck, den Amerika auf mich gemacht hat, ist ein günstiger. Es hat eine bedeutende Gegenwart und, wie auch ich überzeugt bin, eine noch bedeutendere Zukunft. Allerdings sind die amerikanischen Zustände nicht frei von Schattenseiten. [...] Meine Überzeugung ist, dass wir vieles von den Amerikanern lernen können, - gerade so wie sie von uns“ [42]. Und in der „3. Amerikafahrt“, 1905, hieß es: „Die öfters in Europa mündlich und schriftlich geäußerte Ansicht, dass der Amerikanische Ehemann von seiner Frau in hartem Frohndienst gehalten werde, ist gewiß nicht thatsächlich begründet. Es giebt auch in den Vereinigten Staaten äußerst zärtliche Ehen. [...] Ich betrachte die unter dem Sternenbanner sich vollziehende Kultur-Entwicklung von dem Standpunkt des freundlichen Beobachters, der seine eigne schwarzweißrothe Flagge (Anmerkung: Schwarz-Weiß-Rot waren die Farben des Deutschen Kaiserreichs) stets hochhält" [1].

Obwohl „Landratte“, schien er Meer, Küste und maritime Anlagen („Natürlich habe ich, bei meiner Vorliebe für Hafenanlagen, nicht versäumt, den ganzen Hafen von Bombay im Boot zu durchfahren und alles genau in Augenschein zu nehmen“ [47]) besonders geliebt zu haben. Er war sicher kein „Spielertyp“ („Zu den Spielkarten brauche ich meine Zuflucht nicht zu nehmen. Ich verstehe keines der Kartenspiele“ [47]). Von irgendwelchen Hobbies berichtete Hirschberg nicht. Gelegentlich zitierte er in seinen Berichten Goethe (1749-1832), den er, wie William Shakespeare (1564-1616) und Richard Wagner (1813-1883), besonders schätzte. Den „Faust“ konnte er eine Stunde und länger rezitieren, wie er überhaupt lieber selbst redete als zuhörte [6].

Natürlich spielte die Augenheilkunde auch auf den Reisen eine Rolle. Hirschberg besuchte hin und wieder befreundete Augenärzte wie Andreas Anagnostakis (1826-1897) in Athen [49] und Hermann Knapp in New York [1], aber auch andere, heute unbekannte Fachkollegen. In Tokyo traf er mit Dr. Miyashita zusammen [47], der beim ihm in Berlin gehört hatte und vermutlich der „Mijashita“ aus Osaka ist, der gut 25 Jahre später, 1920, die Diskriminierung der deutschen Augenheilkunde nach dem Ersten Weltkrieg in einem offenen Brief anprangern sollte [53]. Es sei in diesem Zusammenhang daran erinnert, dass sich die deutsch-japanischen Kontakte in der Ophthalmologie am Ende des 19. Jahrhunderts recht intensiv entwickelten und Julius Hirschberg nur einer von vielen deutschen Ophthalmologen war, die damals mit japanischen Kollegen in Kontakt standen [54]. Bei seiner letzten Griechenlandreise begleitete ihn der Wiener Ordinarius Ernst Fuchs (18511930) für einige Tage auf einer Wanderung [49]. „Ophthalmologische Konsultationstätigkeit“ hielt Hirschberg, wenn irgendwie möglich, auf Reisen nicht ab, aber er sah „nebenbei“ Kataraktpatienten in Griechenland und Indien, Trachomkranke vor allem in Tunis und Ägypten sowie (oft einseitig erblindete) Pockenkranke in Indien $[45-47,49]$. In Indien stellte er durchaus ordentliche Ergebnisse nach Starstich fest, mokierte sich aber natürlich über einen Inder, der in Berlin sein Unwesen mit Starstich trieb: „Es ist erstaunlich, wie in Berlin erwachsene Menschen einem hergelaufenen, geldgierigen Hinter-Indier sich anvertrauen konnten, während ihnen zahlreiche gelehrte, geübte Wundärzte unentgeltlich zur Verfügung stehen“ [47]. Bemerkenswert ist, dass Hirschberg in seinen Reiseberichten kein Wort über seine eigene Augenklinik in Berlin verlor, und er auch nichts von Depeschen an ihn bezüglich schwieriger oder wichtiger Patienten erzählte, wie das bei Albrecht von Graefe mitunter vorkam [40]. Ganz augenscheinlich funktionierte seine Klinik also während seiner Abwesenheit dank seiner Schüler problemlos. Oskar Fehr und Wilhelm Mühsam waren beide erfahrene Kliniker. Fehr, ab 1900 (bis Ende 1933) Chefarzt der neu gegründeten Augenklinik am VirchowKlinikum, galt dabei als der bessere Wissenschaftler [6].

Hirschberg lebte von seinem „phänomenalen Gedächtnis, das ihm alles je Gelesene und Gehörte zum bleibenden Besitz werden ließ. Dabei war er sich seiner überragenden Fähigkeiten voll bewusst, und Selbstbewusstsein konnte sich gelegentlich wie bei einem homerischen Helden in geradezu naiver Weise äußern“ [4]. Obschon Hirschberg von den Meeren, dem Yellowstone-Park und dem Yose- 
mite-Tal in den USA als „eine der wunderbarsten Gegenden der Erdoberfläche“ und dem Tadj Mahal in Agra/Indien als „schönstem Gebäude Asiens“ schwärmte, so fühlte er sich doch Griechenland, das er mehrfach bereiste, ganz besonders verbunden. Zu den alten Ärzten dort schrieb er 1922: „Noch heute, nach 2300 Jahren, sind die hippokratischen Schriften uns lebendig geblieben; die Stimme jener alten Ärzte klingt noch vernehmlich an unser Ohr. [...] Wenn wir auch die praktische Heilkunde von ihnen nicht erlernen wollen, nicht erlernen können; so möchte ich doch als klinischer Lehrer darauf hinweisen, dass ihre getreue, naive Beobachtung des erkrankten Menschen noch heute mustergültig ist für den angehenden Arzt, und ein Gegengewicht bildet gegen die übergroße Macht des Laboratoriums, und dass die schöne Form ihrer Schriften mehr als einen bloßen Schmuck bedeutet“ [55]. Die „Hellas-Fahrten“ sind eine einzige Liebeserklärung an dieses Land, und so schrieb ein unbekannter Philologe im Literarischen Zentralblatt zu Deutschland von 1910 folgende Rezension dazu: „Julius Hirschberg ist nicht nur ein berühmter Augenarzt, sondern auch ein ausgezeichneter Kenner des klassischen Altertums, seine ,Geschichte der Augenheilkunde im griech. Altertum' ist jedem Philologen als eine wissenschaftliche Leistung ersten Ranges bekannt. Ich habe es (Anmerkung: das Buch „Hellas-Fahrten“) in einem Zuge gelesen und mir dann gesagt: das ist vielleicht das Interessanteste und Beste, was seit langem über Hellas geschrieben worden ist. Besonderen Reiz verleiht den Ausführungen des Verf. die Verknüpfung der Gegenwart mit dem Altertum durch Einflechtung von Zitaten aus der antiken Literatur und allerhand historischer Reminiszenzen“ (Rezension auf der Rückseite von [11]). Eben dadurch war Julius Hirschberg ein Reisender durch Welt und Zeit. In „Um die Erde“ resümierte er im Jain-Tempel auf dem Berg Abu in Indien „Die breitesten und tiefsten Meere habe ich durchschifft, in Sonnengluth und Sturmeswuth, die höchsten Berge habe ich geschaut; ich sah die wunderbarste Ueppigkeit des Pflanzenwuchses, die seltensten Thiere, die merkwürdigsten Menschen, ihre Sitten und Kunst, die herrlichsten und grossartigsten Bauwerke auf der Oberfläche des Planeten: doch nun sehne ich mich nach Hause, zu den Meinen, zu meiner Thätigkeit. [...] Fast ist es mir, wie ein schöner Traum. Aber die wechselnden Bilder stehen lebendig vor meinem Auge.
Mein Herz ist voll Dankbarkeit gegen das neunzehnte Jahrhundert, das die Entfernung vernichtet und solche Reisen ermöglicht hat" [47]. Autos und Flugzeuge waren noch nicht absehbar gewesen.

„Gerade der Geschichtsschreiber weiß am besten, dass viele Arbeiten nur für ihre Zeit einigen Wert besaßen, etliche auch den nicht einmal“ schrieb Hirschberg 1913 in seiner Vorrede zur Festschrift zu seinem 70. Geburtstag [11]. Für seine Reiseberichte, viele seiner Monografien und Publikationen sowie insbesondere für seine „Geschichte“ gilt das nicht. Deren Wert ist zeitlos. Wenn es einen Augenarzt gegeben hat, der Ophthalmologie, Medizin, Naturwissenschaften, Literatur, Philosophie, Archäologie, Geografie, Kunst, Kultur und Geschichte in geradezu idealer, harmonischer Weise miteinander zu verbinden wusste, dann war es Julius Hirschberg. Die Zukunft wird keinen zweiten Hirschberg hervorbringen können.

\section{Danksagung \\ $\nabla$}

Der Beitrag ist all jenen gewidmet, welche die Julius-Hirschberg-Gesellschaft seit ihrer Gründung mit Leben gefüllt haben.

Der Autor hat 30 Jahre in den „Klinischen Monatsblättern“ zu ganz verschiedenen Themen publizieren dürfen. Er dankt dem (ehemaligen) Enke-Verlag und dem Thieme-Verlag sowie den Schriftleitern in dieser Zeit für das entgegengebrachte Vertrauen.

\section{Interessenkonflikt: Nein.}

\section{J. M. Rohrbach}

Department für Augenheilkunde,

Forschungsbereich Geschichte der

Augenheilkunde/Ophthalmopathologisches Labor, Eberhard-Karls-Universität Tübingen

\section{Literatur}

1 Hirschberg J. Meine dritte Amerika-Fahrt. Med. Klinik 1905, Nr. 42: 1056-1061, Nr. 43: 1084-1088, Nr. 45: 1135-1140, Nr. 47: 1191-1196

2 Hirschberg J. Aus jungen Tagen. Berlin: W Junk; 1923

3 Mühsam W. Julius Hirschberg †. Zeitschr Augenheilkd 1925; 55: 289-292

4 Fehr 0 . Julius Hirschberg $†$. Klin Monatsbl Augenheilkd 1925; 74: 497-501

5 Koelbing HM. Julius Hirschberg (1843-1925) als Ophthalmologe und Medizinhistoriker. Klin Monatsbl Augenheilkd 1976; 168: 103-108

6 Jokl A. Julius Hirschberg. Am J Ophthalmol 1959; 48: 329-339
7 Hirschberg J. Die mathematischen Grundlagen der medizinischen Statistik. Leipzig: Veit \& Comp.; 1874

8 Hirschberg J. Ueber die Anwendung der Wahrscheinlichkeitsrechnung auf die therapeutische Statistik. Berliner Klin Wochenschr 1877;14: 297-299

9 Hirschberg J. Dioptrik der Kugelflächen und des Auges. Arch Anat Physiol Wiss Med 1876: 587-621

10 Hirschberg J. Reform der Augenheilkunde, 2. Teil. In: Graefe A, Saemisch T, Hrsg. Handbuch der gesamten Augenheilkunde, 2. Aufl. Berlin: Julius Springer; 1918, 60-68

11 Fehr O, Mühsam W. Julius Hirschberg's ausgewählte Abhandlungen (1868-1912) zu seinem 70. Geburtstage ihm überreicht im Namen seiner Schüler. Leipzig: Veit \& Comp.; 1913

12 Snyder C. Julius Hirschberg, the neglected historian of ophthalmology. Am J Ophthalmol 1981; 91: 664-676

13 Hirschberg J. Erster Bericht über Dr. Hirschberg's Augenklinik. Berliner Klin Wochenschr 1870; 7: 528-531, 540-542, 551553

14 Friedenwald $H$. Obituary notice. Julius Hirschberg. Arch Ophthalmol 1925; 54: 418-422

15 Hirschberg $J$. Ueber das antiseptische Verfahren in der Augenheilkunde. Centralbl Prakt Augenheilkd 1878; 2: 151-152

16 Rohrbach JM. Albrecht von Graefe (18281870) und die Ophthalmopathologie. Klin Monatsbl Augenheilkd 2015 [im Druck] DOI: $10.1055 / \mathrm{s}-0035-1545738$

17 Hirschberg J. Anatomische Untersuchungen über Glioma retinae. Arch Ophthalmol (von Graefe) 1868; 14/II: 30-102

18 Hirschberg J. Der Markschwamm der Netzhaut. Berlin: August Hirschwald; 1869

19 Hirschberg J. Ueber specifische Hornhautentzündung. Centralbl Prakt Augenheilkd 1888; 12 : 216-219 und 254-264

20 Hirschberg $J$. Ein seltener Operationsfall. Centralbl Prakt Augenheilkd 1879; 3: 376381 (Nachdruck der Erstpublikation in der Berliner klinischen Wochenschrift 46/1879)

21 Hirschberg J. Die Magnet-Operation in der Augenheilkunde. Nach eigenen Erfahrungen dargestellt, 2. Auflage. Leipzig: Veit \& Comp.; 1899

22 Hirschberg J. Über die Magnet-Operation in der Augenheilkunde. Centralbl Prakt Augenheilkd 1907; 31: 114-117

23 Hirschberg J. Ueber Messung des Schielgrades und Dosierung der Schieloperation. Centralbl Prakt Augenheilkd 1885; 9: 325327

24 Hirschberg J. Beiträge zur Lehre vom Schielen und von der Schieloperation. Centralbl Prakt Augenheilkd 1886; 10: 5-9

25 Hirschberg J. Einführung in die Augenheilkunde. Leipzig: Georg Thieme, erste Hälfte 1892, zweite Hälfte 1901

26 Hirschberg J. Klinische Beobachtungen aus der Augenheilanstalt. Wien: Wilhelm Braumüller; 1874

27 Hirschberg J. Wörterbuch der Augenheilkunde. Leipzig: Veit \& Comp.; 1887

28 Hirschberg J. Professor A. von Graefe's klinische Vorträge über Augenheilkunde. Berlin: August Hirschwald; 1871 
29 Hirschberg J. Albrecht von Graefe. Heft 7 von Männer der Wissenschaft (Hrsg. J. Ziehen), Leipzig: Wilhelm Weicher; 1906

30 Hirschberg J. Reform der Augenheilkunde, 1. Teil. In: Graefe A, Saemisch T, Hrsg. Handbuch der gesamten Augenheilkunde, 2. Aufl. Berlin: Julius Springer; 1918, 208-314

31 Hirschberg J. Augenkrankheiten und ihre Behandlung. Vorlesungen gehalten an der Universität zu Berlin im Wintersemester 1859-1860 von Prof. Dr. Albrecht von Graefe. Leipzig: Georg Thieme; 1925

32 Hirschberg J. Die Augenheilkunde des Aetius aus Amida. Griechisch und Deutsch. Leipzig: Veit \& Comp.; 1899

33 Hirschberg J, Lippert J. Die Augenheilkunde des Ibn Sina. Aus dem Arabischen übersetzt und erläutert. Leipzig: Veit \& Comp.; 1902

34 Hirschberg J. Ueber die Sprache der Aerzte. Dtsch Med Wochenschr 1892; 18: 215-218

35 Hirschberg J. Zur vergleichenden Ophthalmoskopie. Centralbl Prakt Augenheilkd 1882; 6: 173-177

36 Hirschberg J. Zur Dioptrik und Ophthalmoskopie der Fisch- und Amphibienaugen. Centralbl Prakt Augenheilkd 1882; 6: 504506

37 Rohrbach JM. Jüdische Augenärzte im Nationalsozialismus - eine Gedenkliste. Klin Monatsbl Augenheilkd 2011; 228: 70-83

38 Rohrbach JM. Auge und Tod. Klin Monatsbl Augenheilkd 2014; 231: 73-78

39 Rohrbach JM, Nessmann A, Leitritz MA. „Aus alt mach neu“: Hermann Cohn und das Licht-Konzept zur Myopie-Prävention
1867-2015. Klin Monatsbl Augenheilkd 2015 [im Druck] DOI: 10.1055/s-00351557757

40 Schilp T, Rohrbach JM. Albrecht von Graefe an Frans Cornelis Donders. Briefe 18521870. Essen: Klartext; 2013

41 Jacobson J. Reisebriefe aus Italien und der Schweiz. Nach seinem Tode herausgegeben. Königsberg: Wilhelm Koch; 1893

42 Hirschberg J. Von New York nach San Francisco. Tagebuchblätter. Leipzig: Veit \& Comp.; 1888

43 Hirschberg J. Die Pariser Augenkliniken. Berliner Klin Wochenschr 1876; 13: 625-626

44 Hirschberg J. Die Londoner Augenkliniken. Dtsch Zeitschr Prakt Med 1877; 4: 313-315 (und andere)

45 Hirschberg J. Eine Woche in Tunis. Leipzig: Veit \& Comp.; 1885

46 Hirschberg J. Aegypten. Geschichtliche Studien eines Augenarztes. Leipzig: Georg Thieme; 1890

47 Hirschberg J. Um die Erde - Eine Reisebeschreibung. Leipzig: Georg Thieme; 1894

48 Hirschberg J. Aerztliche Bemerkungen über eine Reise um die Erde. Dtsch Med Wochenschr 1893; 19: 308-310 sowie 1894; 20: 109-111 (und andere)

49 Hirschberg J. Hellas-Fahrten. Leipzig: Veit \& Comp.; 1910

50 Hirschberg J. Für das Vaterland. Centralbl Prakt Augenheilkd 1914; 38: 225

51 Kirisawa $N$. The Komoto library (formerly the Hirschberg library). Jpn J Ophthalmol 1977; 21: 528-532
52 Hirschberg J. Vom Verfasser überreicht. Katalog der Bücher-Sammlung von J. Hirschberg. Berlin; 1901 (ohne Verlagsangabe, wahrscheinlich Eigenverlag)

53 Rohrbach JM. Die deutsche Augenheilkunde am Übergang vom Kaiserreich zur Weimarer Republik (1915-1925). Klin Monatsbl Augenheilkd 2012; 229: 262-271

54 Schmidt $D$. Deutschsprachige Publikationen von Japanern (1886-1941). Klin Monatsbl Augenheilkd 2012; 229: 832-839

55 Hirschberg J. Vorlesungen über Hippokratische Heilkunde. Leipzig: Georg Thieme; 1922

\section{Bibliografie}

DOI http://dx.doi.org/10.1055/s-0035-1557890 Online-publiziert: 13.10.2015

Klin Monatsbl Augenheilkd 2015; 232: 1413-1421 (c) Georg Thieme Verlag KG Stuttgart · New York . ISSN 0023-2165

\section{Korrespondenzadresse}

\section{Prof. Dr. Jens Martin Rohrbach}

Department für Augenheilkunde, Forschungs-

bereich Geschichte der Augenheilkunde/

Ophthalmopathologisches Labor

Eberhard-Karls-Universität Tübingen

Schleichstr. 6-12

72076 Tübingen

Tel.: + 49/(0)7071/2984761

Fax: + 49/(0)7071/294762

Martin.Rohrbach@med.uni-tuebingen.de 